\title{
https://helda.helsinki.fi
}

\section{Light-sample interaction in microsphere enhanced 2D super-resolution imaging}

\section{Maconi, Goran}

SPIE - the international society for optics and photonics 2019

Maconi , G , Kassamakov , I, Vainikka , T , Arstila , T \& Haeggstrom , E 2019 , Light-sample interaction in microsphere enhanced $2 \mathrm{D}$ super-resolution imaging . in $\mathrm{P}$ Lehmann, W Osten \& A A Gonçalves Jr. (eds), Optical Measurement Systems for Industrial Inspection XI . Proceedings of SPIE , vol. 11056 , SPIE - the international society for optics and photonics , Bellingham, WA, Optical Measurement Systems for Industrial Inspection , München , Germany , 24/06/2019 . https://doi.org/10.1117/12.2525489

http://hdl.handle.net/10138/314821

https://doi.org/10.1117/12.2525489

unspecified

acceptedVersion

Downloaded from Helda, University of Helsinki institutional repository.

This is an electronic reprint of the original article.

This reprint may differ from the original in pagination and typographic detail.

Please cite the original version. 


\title{
Light-sample interaction in microsphere enhanced 2D super- resolution imaging \\ Göran Maconi*a, Ivan Kassamakov ${ }^{\mathrm{a}, \mathrm{b}}$, T. Vainikka ${ }^{\mathrm{b}}$, Timo Arstila ${ }^{\mathrm{b}}$, Edward Hæggström ${ }^{\mathrm{a}}$ ${ }^{a}$ University of Helsinki, Helsinki, Finland, banojet Inc., Helsinki, Finland
}

\begin{abstract}
We simulate the image generated by a microsphere residing in contact on top of an exposed Blu-ray disk surface, when observed by a conventional microscope objective. While microsphere lenses have been used to focus light beyond the diffraction limit and to produce super-resolution images, the nature of the light-sample interaction is still under debate. Simulations in related articles predict the characteristics of the photonic nanojet (PNJ) formed by the microsphere, but so far, no data has been published on the image formation in the far-field. For our simulations, we use the open source package Angora and the commercial software RSoft FullWave. Both packages implement the Finite Difference Time Domain (FDTD) approach. Angora permits us to accurately simulate microscope imaging at the diffraction limit. The RSoft FullWave is able to record the steady-state complex electrical and magnetic fields for multiple wavelengths inside the simulation domain. A microsphere is simulated residing on top of a dielectric substrate featuring sub-wavelength surface features. The scattered light is recorded at the edges of the simulation domain and is then used in the near-field to far-field transformation. The light in the far field is then refocused using an idealized objective model, to give us the simulated microscope image. Comparisons between the simulated image and experimentally acquired microscope images verify the accuracy of our model, whereas the simulation data predicts the interaction between the PNJ and the imaged sample. This allows us to isolate and quantify the near-field patterns of light that enable super-resolution imaging, which is important when developing new micro-optical focusing structures.
\end{abstract}

Keywords: Super-resolution imaging, FDTD, simulation, Near-field optics, Photonic nanojet

\section{INTRODUCTION}

Optical microscopy, as well as any wave-based imaging method, is limited in resolution by diffraction. In optics several ways have been devised to circumvent this limit, including confocal microscopy, fluorescence microscopy, and so-called photonic nanojets (PNJs). The ability of microspheres to focus light beyond the diffraction limit was discovered in 2000 , where microspheres on a silicon surface produced sub-wavelength sized irradiation marks [1]. This phenomenon can be produced by microspheres and other micro-optical elements. Experiments [2,3] have shown that these elements can also be used to produce images whose resolution exceeds the classical diffraction limit, hence the popular term "superresolution". Microsphere-enhanced super-resolution imaging is an extension of conventional microscopy that enables subdiffraction-limit resolution while retaining the versatility of classical light microscopes. In this work, we concentrate on super-resolution 2D imaging enhanced by microspheres. We model the focusing and image forming properties of such a system, using the FDTD [4] algorithm, since the mechanism for the light-sample interaction is still unclear, and the role of photonic nanojets in super-resolution imaging is currently under debate.

Our aim is to improve this understanding by simulating the light-sample interaction by using the FDTD approach in combination with Near-Field to Far-Field Transformation (NFFFT) [5] for calculating the image formation. The simulations in conjunction with our experimental data provide detailed predictions about the conditions required for superresolution imaging, as well as predictions about the electromagnetic field giving rise to this phenomenon. In our case, the PNJ-generating structure is a polymer microsphere and the sample is a polymer grating with dimensions comparable to a Blu-ray disk.

Modeling is done using Synopsis' Rsoft FullWAVE, a commercial software package that allows FDTD simulation in 3D and that provides a steady-state analysis of the simulation. Since only the base FDTD package of Rsoft is at our disposal, our simulations are limited to the area of near-field interaction. Therefore, we also use Angora [6], a free FDTD package developed at Northwestern University (Backman Biophotonics Group, Evanston, Illinois, United States) which is built for far-field bioimaging applications.

*goran.maconi@helsinki.fi 


\section{METHODS}

The simulation geometry is based on experimental data obtained with an instrument that allows super-resolution imaging in both 2D and 3D. Melamine formaldehyde microspheres $(\mathrm{d}=11 \mu \mathrm{m}, \mathrm{n}=1.68)$ were placed on a peeled Blu-ray surface, to produce super-resolution images of the grating lines. The system uses an infinity-corrected objective (Nikon CF IC EPI Plan DI) with a magnification of 50X and a numerical aperture of 0.55. The image is recorded by a Hamamatsu Orca Flash 2.8 camera.

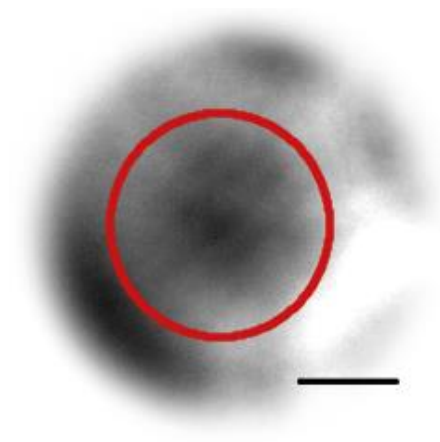

Figure 1. 2D image obtained with our super-resolution instrument, using $11 \mu \mathrm{m}$ melamine formaldehyde microspheres on a peeled Blu-ray disk surface. The size bar is $1 \mu \mathrm{m}$, the field of view is marked by the red circle.

\subsection{Free nanojet simulations}

We simulate the focusing properties of the melamine formaldehyde sphere in the FDTD software Rsoft FullWAVE. This gives us the steady-state complex electrical field in 3D. White light is simulated by a sine-modulated gaussian pulse, that propagates downwards as a plane wave (Fig. 2). For the steady-state calculations, 10 discrete wavelengths in the visible spectrum (400-700 nm, linearly spaced over $1 / \lambda)$ are sampled. When visualizing the electromagnetic fields, the intensities are added together to create a white-light intensity image. The simulation domain was discretized into $20 \mathrm{~nm}$ cubic cells.

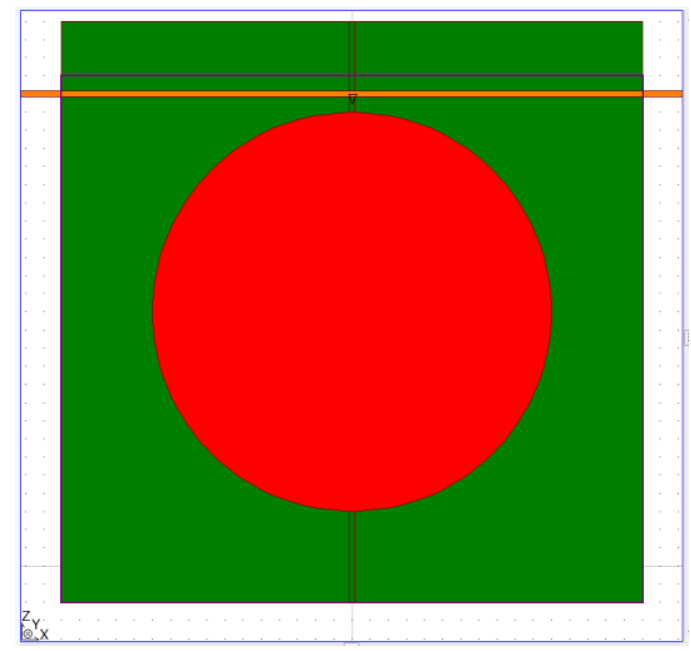

Figure 2. Nanojet simulation domain in Rsoft. The plane wave (orange) traverses downward through the sphere and the electromagnetic field is captured in the xz- and yz-planes (green). The simulation domain boundaries are marked in purple 


\subsection{Far-field imaging simulations}

Far-field imaging was simulated using Angora. The sample target was a polycarbonate $(\mathrm{n}=1.59)$ grating with a pitch of $320 \mathrm{~nm}$ and a groove depth of $100 \mathrm{~nm}$. This is laterally similar to the peeled surface of a Blu-ray disc, but the depth is increased to provide additional contrast for our simulation. To prevent edge artifacts from interfering with the central part of the image, the simulation domain was $40 \times 40 \times 13 \mu \mathrm{m}^{3}$, leaving empty space next to the sphere (Fig. 3). The grating was rotated $15^{\circ}$, to make it easier to identify potential edge artifacts. The spatial resolution in the simulation was $10 \mathrm{~nm}$, which suffices to preserve the detail of the grating surface when discretized. Simulations were run on a supercluster using openMPI-parallelization. The system is excited using a Total-Field/Scattered-Field scheme, where the illumination is separated from the scattered light in the far-field. Like in angora, the scene is illuminated by a plane wave consisting of a sine-modulated gaussian pulse. The far-field scattering is then calculated for 10 discretized wavelengths in the visible spectrum $(400-700 \mathrm{~nm})$.

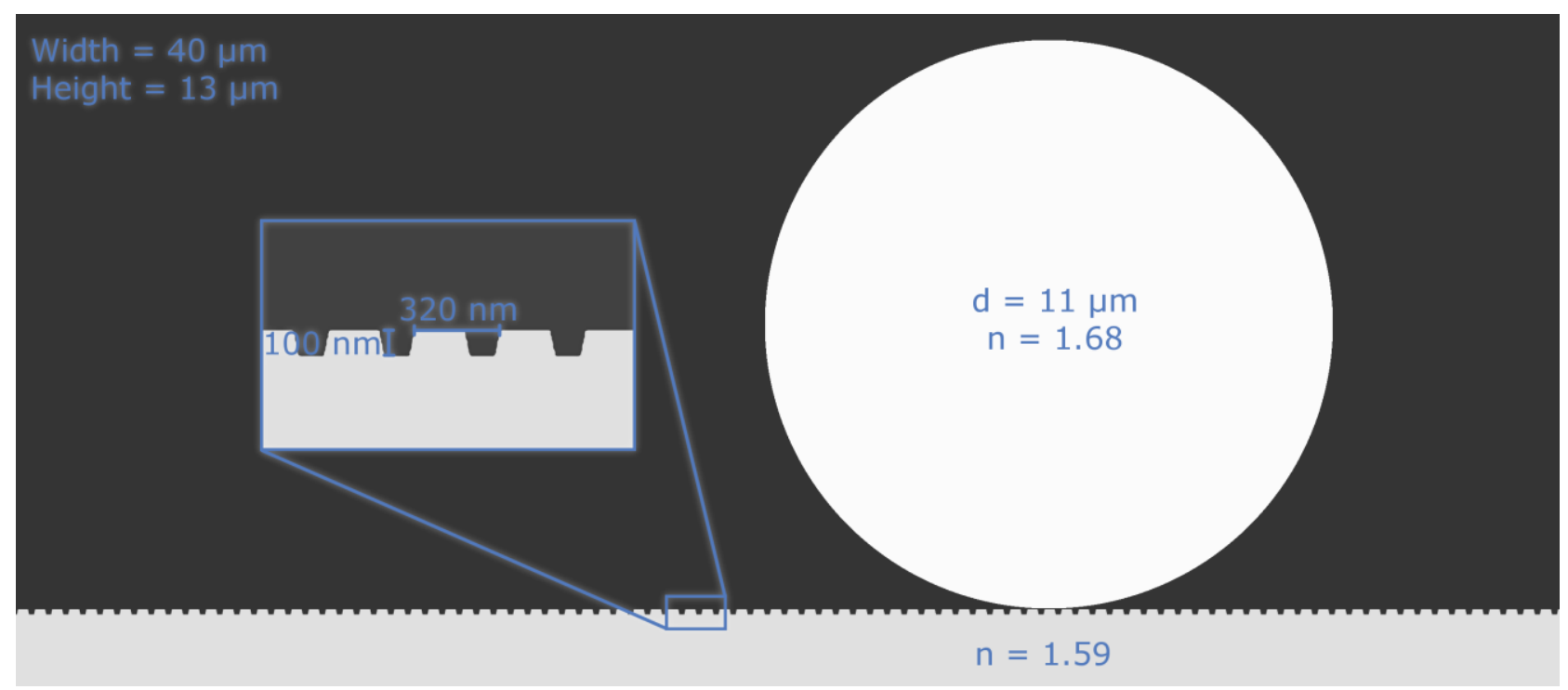

Figure 3. Schematic and xz-cross-section of the microsphere-enhanced imaging simulation in Angora.

\subsection{Ray tracing comparisons}

Since it is relevant for us to what extent the wave-based simulations are comparable to ray optics, 2D imaging of the microsphere residing on the grating was simulated using Monte Carlo path tracing [7]. The sample was illuminated by a directional light, emulating the parallel light illumination of the super-resolution instrument (Fig. 4). The objective was simulated by a virtual camera with a numerical aperture of 0.55 and total field of view (FoV) of $16 \times 16 \mu \mathrm{m}^{2}$, with a resolution of 1024 x 1024 pixels. To find the image plane, the focal point of the objective was moved into the sample until the optimal image was achieved.

\section{RESULTS}

The simulations with Rsoft provide comparability to other PNJ simulations in literature, whereas the far-field imaging of angora provides new information on how the image is formed. Additionally, the raytracing simulations show to what extent the system can be modeled with classical optics. 


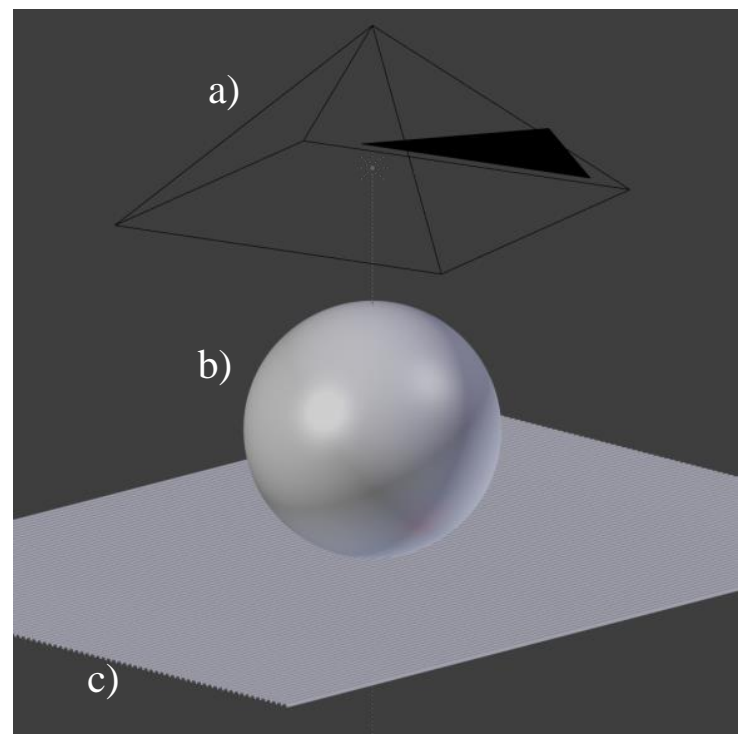

Figure 4. Simulation setup for Monte Carlo path tracing. a) Virtual camera. b) Microsphere $(\mathrm{n}=1.68)$. c) Grating.

\subsection{Free nanojet simulations}

Simulation results from Rsoft FullWAVE show that the sphere focuses white light into a PNJ, i.e. the Full Width at Half Maximum (FWHM) of the focus is smaller than what is described by the Abbe diffraction limit. The PNJ maximum is at a distance of $160 \mathrm{~nm}$ from the bottom surface of the sphere and the FWHM is $0.41 \mu \mathrm{m}$ (Fig. 5).

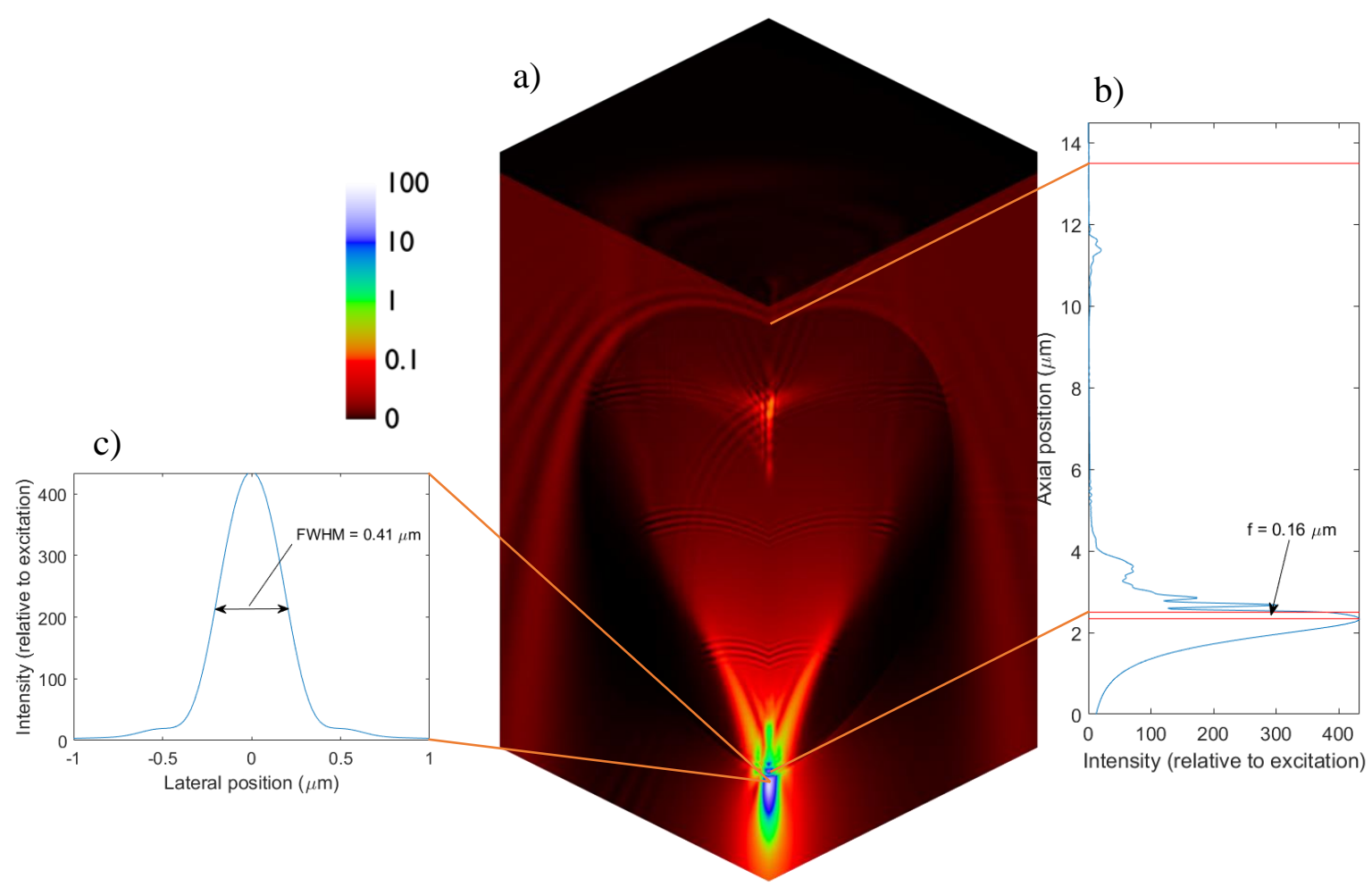

Figure 5. a) Quarter-section of the FDTD simulation domain, showing steady-state white-light intensity at the three crosssections. b) Intensity profile of the central axis of the simulation. c) Lateral profile of the focus 


\subsection{Far-field imaging simulations}

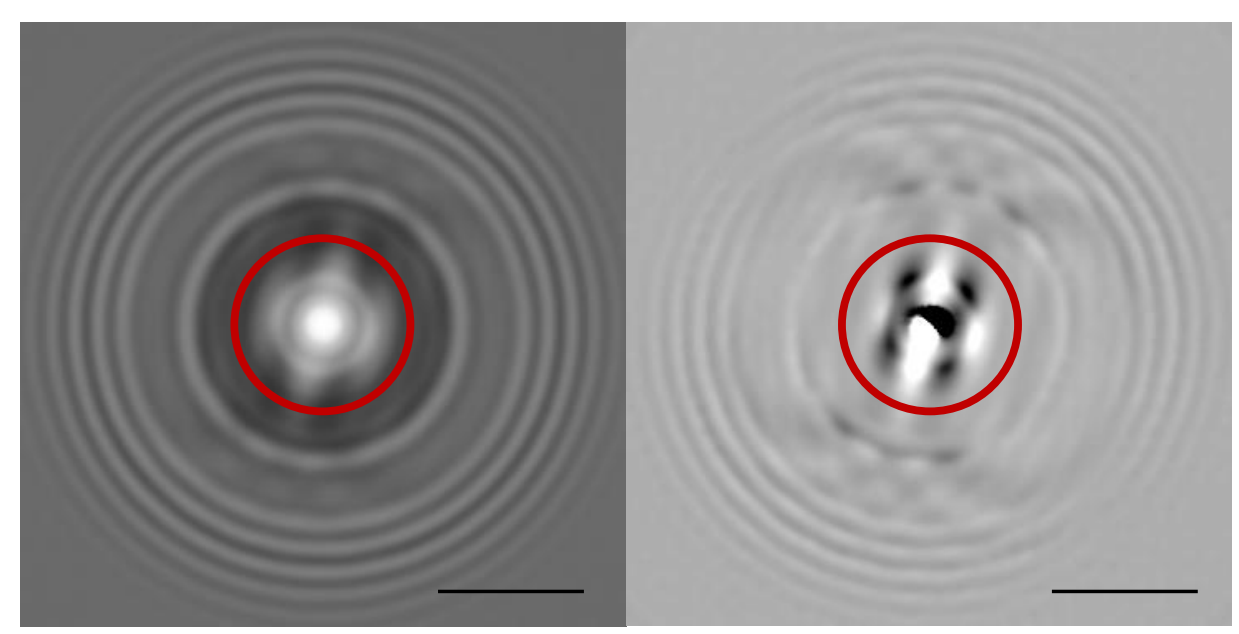

Figure 6. Focused simulated far-field image, the virtual objective is focused through the microsphere. Left: Best focus, unfiltered. Right: Rotationally symmetrical patterns removed. The size bar is $1 \mu \mathrm{m}$ and the FoV, obtained with plane wave illumination is marked in red. The FoV only spans 3 grating lines, due to the narrow illumination

The far-field light scattering data from Angora was refocused using a simulated ideal objective with numerical aperture of 0.55. Since the microsphere produces a virtual image, the focus of the camera was scanned beneath the grating surface until the sharpest image was found. The best image was found at a focal depth of $11 \mu \mathrm{m}$ beyond the surface (Fig. 6). To reduce the contribution of light scattered from the microsphere itself, the rotationally symmetric parts of the light were subtracted from the image, amplifying the light from the sample. The grating lines at $15^{\circ}$ tilt are clearly visible through the sphere, although the FoV is very limited due to the illumination being a perfect plane wave.

\subsection{Ray tracing comparisons}

Using Monte Carlo path tracing, we were able to compare the difference in illumination and FoV between multidirectional and plane wave illumination. The multidirectional illumination was produced by a directional light with a spread of 100 $\mathrm{mrad}$, whereas the plane wave was approximated by narrowing the spread to $10 \mathrm{mrad}$. Zero spread is not allowed by the path tracing algorithm,
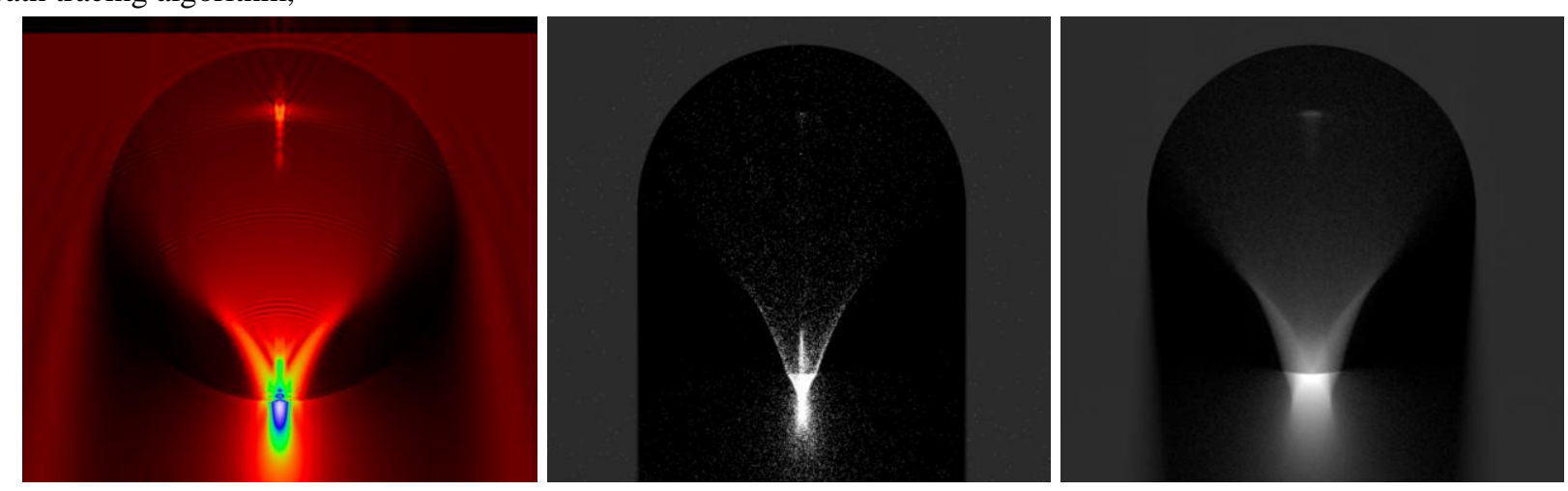

Figure 7. Comparison of simulated PNJs. Left: FDTD simulation of PNJ from a plane wave. Center: Ray traced PNJ from a directional light with low spread. Right: Ray traced simulation of multidirectional illumination focused through the microsphere 
since photons are traced from the camera to the light source. The focus of the narrow light through the sphere is roughly comparable to the FDTD simulations, obviously disregarding wave-based effects, whereas the multidirectional illumination results in a wider even distribution of light at the focal plane (Fig. 7).

The distribution of light at the focus affects the FoV of the image. Using ray tracing, the grating was imaged through the sphere at different spread of the directional light (Fig. 8). At $100 \mathrm{mrad}$, five lines are visible, which is comparable to our experimental results, whereas at $10 \mathrm{mrad}$, three lines can be seen, which corresponds to our FDTD simulation results. The narrow spread results in the characteristic central hotspot, due to the light reflecting straight back from the sphere.
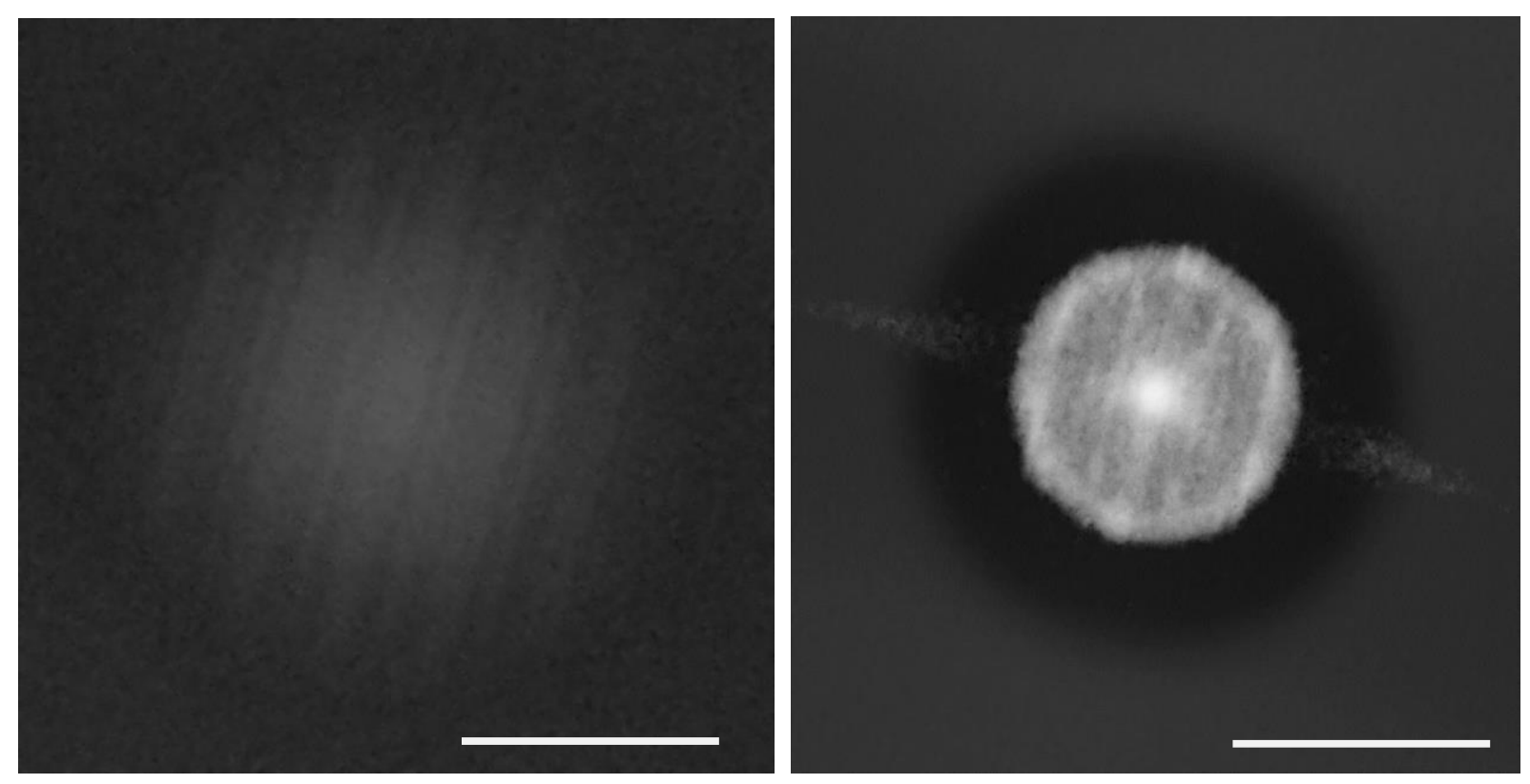

Figure 8. Ray traced simulation of grating imaged through the microsphere Left: Multidirectional illumination. Right: Narrow directional light. The size bar is $1 \mu \mathrm{m}$

\section{CONCLUSIONS}

We simulated 2D imaging beyond the diffraction limit using FDTD software. This allows us to model the light-sample interaction in the near-field of the PNJ generating structure. Due to the computationally intensive nature of simulating multidirectional illumination, we are currently limited to plane wave illumination of our simulated images, resulting in a narrow field of view. This paves the way for FDTD simulation to be used as a tool for developing new and improved PNJ generating structures for super-resolution imaging. Since the phase information is preserved through the far-field imaging process, our next step is to simulate 3D super-resolution imaging using interferometric objectives.

\section{ACKNOWLEDGEMENTS}

We are immensely grateful towards Ms Aya Eid of Northwestern University, for her technical assistance and thoughtful input regarding our use of the Angora software. Thanks to her efforts and expert knowledge, we were able to use Angora to its full potential and correct several issues in our simulations. 


\section{REFERENCES}

[1] Lu et al., "Laser Writing of a Subwavelength Structure on Silicon" (100), Surfaces with Particle-Enhanced Optical Irradiation, Journal of Experimental and Theoretical Physics Letters, (2000).

[2] Lee, Ju Young, et al., "Near-field focusing and magnification through self-assembled nanoscale spherical lenses." Nature 460.7254, 498 (2009).

[3] Guo, Minglei, et al. "Imaging of sub-surface nanostructures by dielectric planer cavity coupled microsphere lens." Optics Communications 383, 153-158 (2017).

[4] Taflove, Allen. "Application of the finite-difference time-domain method to sinusoidal steady-state electromagnetic-penetration problems." IEEE Transactions on electromagnetic compatibility 3, 191-202 (1980).

[5] Çapoğlu, İlker R., et al. "The microscope in a computer: Image synthesis from three-dimensional full-vector solutions of Maxwell's equations at the nanometer scale." Progress in Optics. Vol. 57. Elsevier, 1-91 (2012).

[6] Çapoğlu, İlker R., Allen Taflove, and Vadim Backman. "Angora: A free software package for finite-difference time-domain electromagnetic simulation." IEEE Antennas and Propagation Magazine 55.4, 80-93 (2013).

[7] Veach, Eric, and Leonidas J. Guibas. "Optimally combining sampling techniques for Monte Carlo rendering." In Proceedings of the 22nd annual conference on Computer graphics and interactive techniques, pp. 419-428. ACM (1995). 